Research report

\title{
Influence of imagined posture and imagery modality on corticospinal excitability
}

\author{
Alissa D. Fourkas ${ }^{\mathrm{a}, *}$, Silvio Ionta ${ }^{\mathrm{a}, 1}$, Salvatore M. Aglioti ${ }^{\mathrm{a}, \mathrm{b}, 2}$ \\ a Department of Psychology, University of Rome "La Sapienza” via dei Marsi 78, 00185 Rome, Italy \\ ${ }^{\mathrm{b}}$ IRCCS, Foundation Santa Lucia, Via Ardeatina, 306, 00179 Rome, Italy
}

Received 4 August 2005; received in revised form 20 October 2005; accepted 24 October 2005

Available online 28 November 2005

\begin{abstract}
Single pulse transcranial magnetic stimulation (TMS) was used to test the assumption that kinesthetic imagery of action is more 'motor' than visual imagery of action. We assessed corticospinal excitability during motor imagery of a thumb-palm opposition movement by recording potentials evoked by TMS from two hand muscles that would (opponens pollicis, OP, target) or would not (abductor digiti minimi, ADM, control) be activated during actual performance of the very same movement. Participants were asked to imagine the thumb-palm opposition movement while maintaining first person imagery that was either purely visual or predominately kinesthetic. The motor imagery task was performed in two conditions in which the imagined and the actual hand could be either congruent or incongruent. Facilitation of potentials recorded from OP was higher during imagery carried out in mentally congruent than incongruent postures. This effect was largely due to lack of excitability recorded during incongruent kinesthetic imagery, which was indistinguishable from baseline imagery of the static hand. All other conditions differed from static imagery regardless of position. No significant effects were found in a control muscle (ADM) thus indicating that the effect was not related to spatial coding. Subjective reports obtained after the experiment indicate that the results cannot be ascribed to qualitative differences in the imagery experienced. For relatively simple motor tasks requiring no 'expertise' we found no detectable difference in the motor cortex due to imagery modality.
\end{abstract}

(C) 2005 Elsevier B.V. All rights reserved.

Keywords: Motor imagery; Kinesthetic imagery; First person imagery; Transcranial magnetic stimulation; Mental postural incongruency

\section{Introduction}

Kinesthetic imagery is frequently considered more 'motor' than visual imagery of the same task. This is surprising since motor behavior is highly influenced by visual information. The influence of visual imagery, or more precisely visuospatial imagery, on motor control has been behaviorally demonstrated in reach-grasp [13], endpoint reproduction [25] and locomotion [51] tasks. Although neuroimaging studies indicate that the neural underpinnings of motor imagery and motor execution are similar, differences across studies in reported instructional details and subjective experience $[28,40,42,43,50]$ make it dif-

\footnotetext{
* Corresponding author. Tel.: +3906 49917635; fax: +39 0649917635.

E-mail address: alissa.fourkas@uniroma1.it (A.D. Fourkas).

1 Present address: Department of Clinical Sciences and Bio-imaging, University of Chieti “G. D'Annunzio", Via dei Vestini 31, 66013 Chieti, Italy.

Tel.: +3908713556918

${ }^{2}$ Tel.: +39 0651501509 ; fax: +390651501366.
}

ficult to discern what imagery modality was measured. Recent brain imaging studies indicate activation of a parietal-frontal circuit involving the superior parietal (BA 7), premotor and supplementary motor areas during kinesthetic imagery $[14,49]$ and external visual imagery of oneself acting (i.e. the subject was the agent of the action but the spatial coding was not egocentric) [49]. Activation of somatosensory areas during kinesthetic imagery has not been convincingly documented $[28,33,40,43]$.

The relation between body schema (the representation of the body in the mind) and motor imagery (the mental representation of the body moving) reveals a role for both visual and kinesthetic components in the internal image of a limb. Body schema clearly incorporates both visual and proprioceptive information but under some circumstances one modality dominates over the other (e.g. in the congenitally blind). Visual components are suggested by the similar, albeit less intense, neural activation patterns evident in the primary motor cortex for phantom and non-phantom limbs during visual imagery of a motor task 
[9]. Phantom pain can be alleviated through visual imagery of the body part relaxing [34]. Moreover, chronically hemiplegic patients manifest similar levels of response accuracy in paralyzed and non-paralyzed limbs during implicit motor imagery tasks (e.g. grip selection and rotation of hands) [26]. Thus, the mental representation of the limb needs not be constrained by proprioceptive information; while long term memory may have been accessed for kinesthetic information, this information would contradict the ongoing experience of the paralyzed limb, introducing conflicting signals, and presumably errors, into the motor system.

The influence of proprioceptive inflow on the central representation of body schema is continuous and can induce proprioceptive dominance [46]. Patients have longer reaction times when the hand, which must be mentally rotated, corresponds to the limb in pain [45], in agreement with evidence for anatomical 'constraints' during mental rotation of body parts [39]. Inflow in this context refers to initial sensory information conveying sensation and proprioception [44] rather than muscular inflow [22,23]. The role of muscular inflow is implicit in psychoneuromuscular theory [32], which derives support from research showing increased muscle activation during internal (kinesthetic) imagery [16]. Lutz [29] provides strong evidence that this activity is a by-product of the central generation of a motor image not meaningfully related to skill acquisition or retention, i.e. the pattern of activation during imagery is random and does not match the pattern (e.g. triphasic) recorded during actual performance of the same motor act. There is, in fact, little evidence that peripheral activity is required during motor imagery. Modulation of $\mathrm{F}$ wave amplitude is equivocal $[10,41,52,53]$ while the $\mathrm{H}$ reflex tends to be inactive. Although Bonnet et al. [3] reported $\mathrm{H}$ reflex modulation, the imagery and execution trials were interspersed which may have contributed to the accompanying background EMG activity. Investigations where imagery trials formed a separate block [1] or were no execution trials were performed $[27,21]$ report no change in $\mathrm{H}$ reflex amplitude. Increased $\mathrm{H}$ reflex amplitude is due to practice effects [17].

We compare kinesthetic and first person visual imagery of a thumb-palm opposition task. The task involves more motor than abstract cognitive processes and theoretically should be more closely associated with kinesthetic imagery [cf. 11]. Imagined position of the hand was manipulated to assess whether modulation of motor evoked potentials is strictly due to imagined action of the target muscle, or to spatial information for the target muscle; on half the trials the mental representation of the control muscle (not involved in the action) was 'in view' and more medial (imagined palm up) and half the trials 'out of view' and more lateral (imagined palm down). The control muscle was used as the optimal scalp position in order to maximize the possibility of detecting modulation. Baseline imagery measured the corticospinal excitability accompanying the general representation of the hand: participants imagined their static hand fully congruent with the actual pronated hand; a supinated position was not used due to difficulty maintaining background electromyographic silence.

\section{Method and materials}

\subsection{Participants}

Thirteen participants (age 23-29; five female) participated. All were right handed [4], and neurologically healthy, without psychiatric or other medical disorders, and without any contraindications to transcranial magnetic stimulation (TMS) [cf. 56]. The protocol was approved by the local ethical committee and the research conducted in accordance with the 1964 Declaration of Helsinki. Written informed consent was obtained prior to participation.

\subsection{Electromyography $(E M G)$}

Surface $\mathrm{Ag}-\mathrm{AgCl}$ cup electrodes (1-cm-diameter) were placed over the opponens pollicis (OP) and abductor digiti minimi (ADM) of the right hand by using a belly-tendon montage. Recordings were made using a CED Power 1401 (Cambridge Electronic Design Ltd., Cambridge, UK) connected to an Isolated Patient Amplifier System Model D360 (Digitimer Ltd., Hertfordshire, UK), and interfaced with CED Spike 2 software. The second order Butterworth filter was set between 20 and $2500 \mathrm{~Hz}$ (10 kHz sampling rate). Signals were displayed at a gain of 1000. Auditory feedback of the EMG signals was used to help participants maintain voluntary muscle relaxation during electrophysiological preparation.

\section{3. $T M S$}

Focal TMS was performed with a figure eight shaped stimulation coil (outer diameter of each wing $70 \mathrm{~mm}$ ), connected to a Magstim 200 Mono Pulse (Magstim Whitland, Dyfed, UK), over the left primary motor cortex. The optimal scalp position (OSP) for eliciting MEPs in ADM was found by moving the coil in steps of $1 \mathrm{~cm}$ until the largest MEP was found and then marked with a pen. The coil was held tangential to the scalp with the handle pointing backward and laterally at approximately $45^{\circ}$ from the midline. Resting motor threshold (rMT) was defined as the lowest stimulus intensity to evoke at least 5 out of 10 motor evoked potentials (MEPs) with an amplitude of at least $50 \mu \mathrm{V}$ [37] in both muscles. Stimulus intensity was kept at $20 \%$ above rMT during data collection.

\subsection{Procedure}

Participants were seated comfortably, with their right arm and hand lying pronated on a pillow placed on their lap. Throughout data collection the participants' actual hand was always lying pronated on the pillow. Participants imagined performing thumb-palm opposition (see Fig. 1) in four blocks: visual imagery or kinesthetic imagery, with the imagined palm up (incongruent with actual hand posture) or imagined palm down (congruent with actual hand posture).

Prior to each condition, participants watched their hand as they physically performed the movement for $10 \mathrm{~s}$ with the palm up or palm down (as appropriate to the condition), making note either of the feel or the appearance of the movement (as appropriate to the condition). Participants were told to adopt a constant frequency, and to use the same frequency for their imagined movement. Four movement imagery blocks were performed. To avoid inducing a confound by switching back and forth between modalities, half the participants performed the two kinesthetic imagery conditions first and then the visual imagery conditions, while the other half were assigned the reverse order. Static (baseline) imagery was performed at both the beginning (first block) and end (last block) of the experiment. Participants closed their eyes during imagery.

Each block contained 18 trials. Approximately 5 min elapsed when imagery modality was changed in order to minimize crossover effects while maintaining participant motivation. Written instructions were supplied prior to each condition (see Appendix A, for examples). It was explicitly instructed that the imagery must use a first person perspective with egocentric coding. The instructions for static imagery were for participants to imagine their right hand lying on the pillow and that their imagined hand was completely stationary. Visual imagery instructions stated that the image should contain only the visual information that was available when the movement was actually performed, and that imagining the feeling of the movement should be avoided. The kinesthetic imagery instructions were to imagine the sensations - in particular the stretch and muscle 


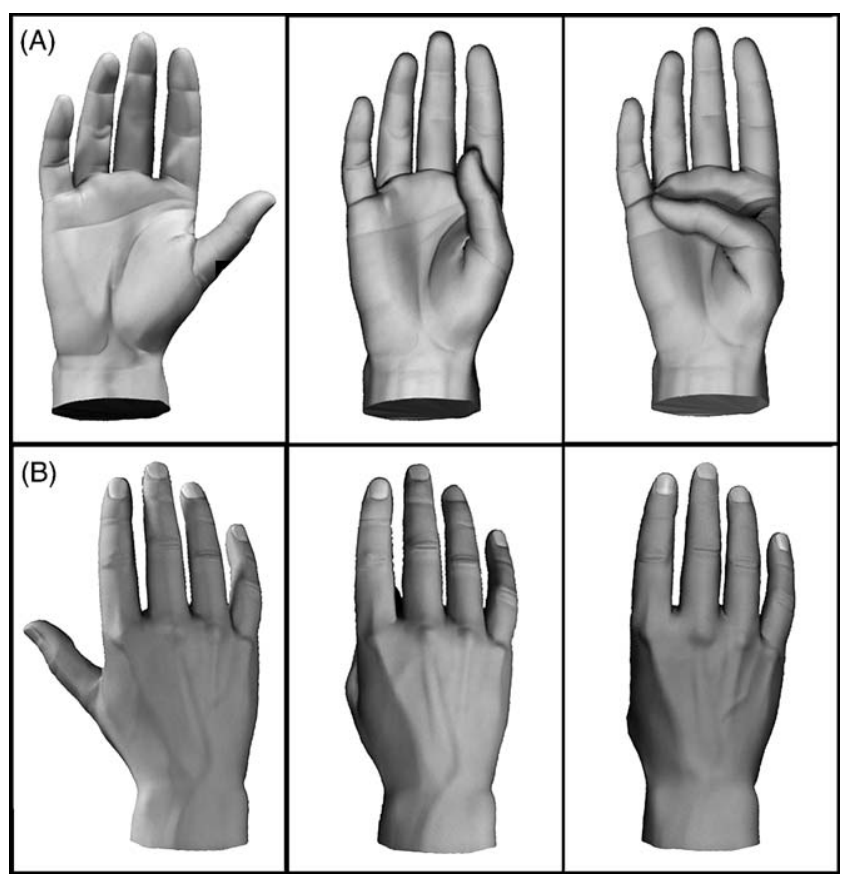

Fig. 1. The movement which participants imagined is illustrated. Upper panels A: thumb-palm opposition was imagined to occur with the palm up (incongruent with actual hand posture) and lower panels B: thumb opposition was imagined to occur with the palm down (congruent with actual hand posture).

tension - that had been present when the movement was actually performed (due to concerns that prohibiting visual information would be too difficult to fully comply with, strong emphasis was placed on the kinesthetic instructions and no mention made of visual information).

Trials began with a computer beep, indicating that the participant should begin performing imagery and initiating EMG recording. To avoid a priming effect, a variable interval of 3-3.5 s elapsed between the beep and the TMS pulse. In the movement conditions task compliance was externally monitored by having participants report the direction of the imagined movement (left/right) when the pulse was delivered. EMG data were recorded for another $0.5 \mathrm{~s}$. A rest period ( $7 \mathrm{~s}$ ) elapsed before the next trial (time between pulses, 10.5-11 s). The inclusion of the rest periods was desirable in order to reduce mental fatigue and attentional lapses. The choice of interstimulus interval was based on research demonstrating no change in corticospinal excitability with repetitive TMS at $0.1 \mathrm{~Hz}$ for $1 \mathrm{~h} \mathrm{[5].}$

\subsection{Post experimental manipulation check}

Participants provided written descriptions of their imagery and rated a series of statements on Likert-type scales for each condition. The statement related to first person imagery controlled for first-third person perspective switching and that the spatial coding was internal (egocentric) rather than external of self. A statement controlled whether kinaesthetic imagery was used during the baseline and visual imagery conditions. The difficulty in forming images was assessed with statements similar to those on the imagery questionnaire. Four aspects of image quality were rated: clarity and vividness of the visual imagery; imagined muscular tension; imagined stretch; and controllability (including maintenance of movement frequency).

\subsection{Imagery questionnaire}

Participants completed an Italian version of the Revised Movement Imagery Questionnaire (MIQr). The MIQr [19] measures the difficulty of forming visual and kinesthetic images of physical movements (e.g. jumping) with a Likert-type scale. The visual imagery instructions do not specify a first or third person perspective. The scale on our Italian version is reversed from the original, with a better image indicated by a lower score $(1=$ very easy; $7=$ very difficult $)$. The Italian version has a Spearman-Brown split-half coefficient of reliability $=0.90$, and Cronbach alpha measure of internal consistency for the visual subscale $=0.79$ and kinesthetic subscale $=0.89$.

\subsection{Subjective experience}

Task compliance was high, as assessed via immediate verbal responses of imagined movement direction after the TMS pulse. Participants kept to a first person perspective in egocentric space. Baseline imagery was clear and vivid, although the lack of activity was mentally fatiguing. One subject indicated that maintaining a purely static image was difficult due to the TMS pulse 'interrupting' the imagery. Two participants reported kinesthetic imagery in the baselines; the feedback indicated that this was due to difficulty distinguishing a kinesthetic image from sensations induced by the TMS pulse. Two participants reported a kinesthetic component during the visual palm up condition and were replaced in the data set. One (retained) subject reported mentally 'severing' her arm to prevent kinesthetic imagery during the visual imagery conditions. In the palm down conditions, participants accommodated the physical contact of the hand with the pillow by: raising their imagined hand; the imagined thumb 'moved through' the pillow, or the pillow was absent from the image. Image quality was typically assessed with positive ratings (Table 1).

\subsection{Data handling}

MEPs were analysed off-line. The absence of background EMG activity was confirmed through visual inspection of the data; trials with background activity

Table 1

Qualitative summary (median response) of the manipulation check

\begin{tabular}{|c|c|c|c|c|c|}
\hline & \multicolumn{2}{|l|}{ Kinesthetic } & \multicolumn{2}{|l|}{ Visual } & \multirow{2}{*}{$\begin{array}{l}\text { Baseline } \\
\text { Down }\end{array}$} \\
\hline & Up & Down & $\mathrm{Up}$ & Down & \\
\hline First & Completely agree & Agree & Agree & Agree & Agree \\
\hline Control & Agree & Agree & Agree & Agree & Agree \\
\hline Kinesthetic & Fairly easy & Fairly easy & & & \\
\hline Tension & Fairly strong & Fairly strong & & & \\
\hline Stretch & Fairly strong & Fairly strong & & & \\
\hline Visual & & & Fairly easy & Fairly easy & \\
\hline Clear/vivid & & & Fairly clear & Fairly clear & Fairly clear \\
\hline
\end{tabular}




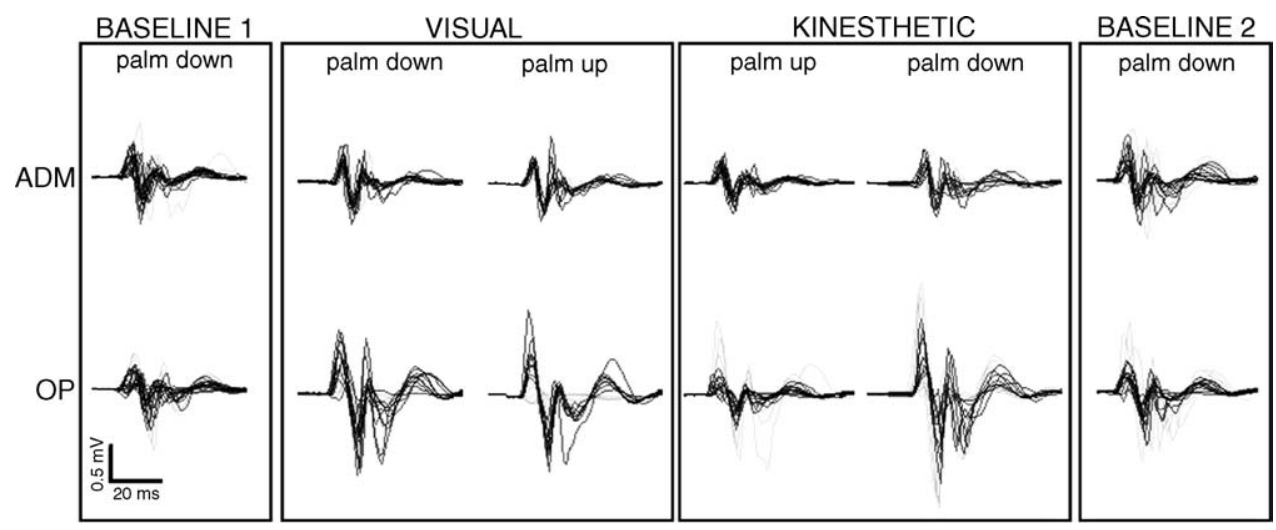

Fig. 2. Raw MEPs recorded in ADM and OP for one representative participant. Data from the 18 trials in each block are superimposed, with their mean values emphasized.

within $100 \mathrm{~ms}$ of the pulse, where the MEP amplitude was difficult to clearly distinguished from background EMG activity $(<90 \mu \mathrm{V})$, or on which movement was observed were discarded. Outliers ( \pm 2S.D.) were identified for each muscle in each condition and the data removed for both muscles. Between 6 and 20 trials were rejected for each participant. Peak-to-peak millivolt $(\mathrm{mV})$ amplitude was calculated using CED Spike 2 software. Raw MEP data from one representative participant are illustrated in Fig. 2. Data were normalized (natural $\log +1)$ to address non-normality resulting from positive skew [35].

Effect size provides an additional index of magnitude of a treatment effect. In repeated measures designs the index is biased by the correlation between two items: for $r>0.5$ a repeated measure effect size will be larger than that for independent groups, and vice versa [31]. We report effect sizes calculated using a modification of the Cohen $d$ statistic to eliminate the bias: $t[2(1-r) / n]^{1 / 2}$, where $t$ is the $t$ statistic for correlated samples, and $r$ is the correlation across pairs of means [8]. The $d$ statistic is interpreted as the number of standard deviations between two means, where 'medium' $d=0.5$ (apparent to the discernable observer), 'small' $d=0.2$ (clearly smaller than medium but not trivial) and 'large' $d=0.8$ (clearly larger than medium) [7].

Responses on the manipulation check were converted to numeric values. These ordinal values were analysed with non-parametric Friedman ANOVAs and Wilcoxon matched-pairs tests [24]. To compare our participants with those in other published research, parametric analysis was used to compare the MIQr subscales.

\section{Results}

\subsection{Baseline}

MEPs in the first and second baseline did not differ in OP, $t(12)=0.92, p=0.38$ (first $M=0.45$, S.D. $=0.23$; second $0.49,0.16$ ) or in ADM, $t(12)=1.23, p=0.24$ (first $M=0.47$, S.D. $=0.23$; second $0.52,0.24)$. The baselines were collapsed and averages obtained.

\subsection{Analysis of MEP data}

Repeated measures ANOVA (four imagined movement conditions and average baseline: Table 2 ) detected no modulation in the control muscle ADM, $F(4,48)=0.86, p=0.49$. In contrast, the target muscle OP was modulated $F(4,48)=5.94, p=0.0006$ (Fig. 3). Newman-Keuls post hoc found that excitability during static imagery was lower than during three of the movement imagery conditions: visual palm down $p=0.002$ (effect
Table 2

Natural log mean (and standard deviation) of MEP peak-to-peak amplitude (mV) in abductor digiti minimi (ADM; control muscle) and opponens pollicis (OP; target muscle)

\begin{tabular}{lllllll}
\hline & \multicolumn{2}{l}{ Kinesthetic Imagery } & & \multicolumn{2}{l}{ Visual Imagery } & \multirow{2}{*}{ Baseline } \\
\cline { 2 - 3 } & Up & Down & & Up & Down & Down \\
\hline ADM & $0.54(0.26)$ & $0.54(0.23)$ & & $0.53(0.25)$ & $0.57(0.23)$ & $0.50(0.22)$ \\
OP & $0.51(0.22)$ & $0.61(0.21)$ & & $0.60(0.24)$ & $0.65(0.30)$ & $0.48(0.18)$ \\
\hline
\end{tabular}

size $d=0.58)$, kinesthetic palm down $p=0.010(d=0.68)$ and visual palm up $p=0.018(d=0.51)$; moreover, excitability during kinesthetic palm up was lower than during visual palm down $p=0.011(d=0.49)$, kinesthetic palm down $p=0.048(d=0.47)$ and visual palm up $p=0.052(d=0.36)$.

Paired $t$-tests indicated no overall difference between imagery modality, $t(12)=1.65, p=0.12$ (visual imagery $M=0.56$, S.D. $=0.21$; kinesthetic imagery $0.62,0.26$ ). A difference between position was obtained, $t(12)=3.09, p=0.009$ (effect size $d=0.33$ ); higher levels of facilitation were recorded in conditions during which the imagined palm position was congruent with the actual hand position (palm down $M=0.63$, S.D. $=0.24$; palm up $0.55,0.23)$.

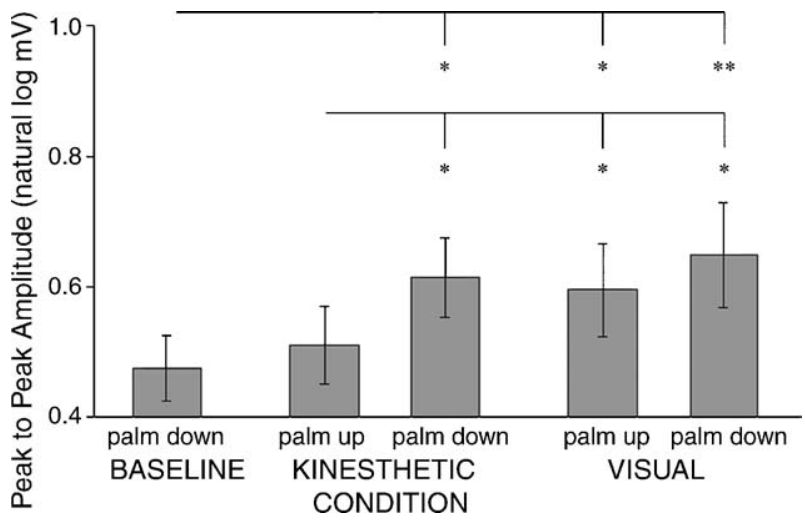

Fig. 3. MEP amplitudes (mean and standard error) in the opponens pollicis. Newman-Keuls post hoc; ${ }^{*} p<0.05,{ }^{* * *} p=0.002$. 


\subsection{Subjective data}

Analyses indicate that the perceived quality of the imagery consciously manipulated was similar across conditions. Friedman ANOVAs (five conditions) for the statements that the imagery was first person egocentric, $\chi^{2}($ d.f. $=4)=7.7, p<0.10$ and controllable, $\chi^{2}$ (d.f. $\left.=4\right)=8.7, p<0.07$, failed to reach significance, as did the statement that the imagery was clear and vivid, Friedman ANOVA (three conditions) $\chi^{2}($ d.f. $=2)=2.0$, $p<0.37$. Wilcoxon matched-pairs found no difference between ratings of imagined tension $(T=9, p=0.21)$, imagined stretch $(T=4, p=0.72)$, difficulty in forming kinesthetic images $(T=14$, $p=1.00)$ or difficulty in forming visual images $(T=1.5$, $p=0.72)$.

\subsection{Imagery questionnaire}

Participants considered it "fairly easy" to form motor images using either modality (visual $M=10$, kinesthetic 12 ); reflecting the scale back to the original direction (a better image indicated by a higher score), participants ratings (visual 22, kinesthetic 20) are similar to those reported elsewhere [19,29]. Visual imagery was considered "easier", $t(13)=2.51, p=0.03$, in agreement with previous research [2,19]. This suggests that our sample is similar to the neurologically healthy population sampled in motor imagery studies.

\section{Discussion}

We found no difference between first person visual and kinesthetic imagery of movement per se, indicating that the effect of both imagery modalities is comparable, at least for simple motor tasks with which the person has no particular 'expertise'. This result was found in spite of explicit instructions prior to each condition (written and physical practice) to concentrate on specific visual or kinesthetic information, and subjective reports which generally indicated good quality imagery. An effect of imagined hand position was found in the opponens pollicis, with higher levels of facilitation recorded when the position of the imagined hand was congruent with the actual hand position. When the imagined hand posture was incongruent with the actual hand a difference between imagery modality was obtained; a visual image of the action leads to an increase in corticospinal excitability while kinesthetic imagery was statistically indistinguishable from static imagery of the hand. Results from the ADM muscle suggest this latter effect was not due to spatial coding; the lack of modulation in ADM suggests that placing the imagined hand in a posture where the muscle being monitored is 'in view' and more medial does not, in itself, lead to changes in MEP amplitude.

We interpret the position effect as evidence that continuous proprioceptive information from the actual hand introduced an informational conflict into the motor system during imagery. This conflict negatively influenced motor imagery when the imagery was kinesthetic. Notably, the optimal scalp position was optimized over a muscle not involved in the movement (ADM), which should have minimized our ability to detect modulation [cf. 12]. Our data agrees with research on imagined finger movements (imagery modality actually used not reported) by Vargas et al. [55]; while their manipulation focused on physical posture and ours on imagined posture, in both cases higher levels of excitability were found with compatible postures. Shenton et al. [46] also reports a congruency effect in that hand and stimuli congruency induced faster response times than incongruency, with the effect largely due to proprioceptive information, i.e. biomechanical constraints.

The subjective reports indicate that in all conditions participants found the imagery reasonably easy to perform and agreed the imagined movement could be controlled. Thus, there appears to be disagreement between the conscious experience of the motor imagery and objective measure of MEP amplitude, mimicking findings involving real movement. Visual and proprioceptive information during pointing, for example, are unavailable for conscious report, supporting the notion that online (dorsal stream) control is not conscious [6]. Hence, it may be notable that first person imagery may utilize a parietal-frontal circuit $[38,47,48]$. However, among participants who spontaneously 'ranked' the relative difficulty of the four movement conditions a consistent pattern occurred: visual palm down imagery was considered easiest, followed by visual palm up, kinesthetic palm down and kinesthetic palm up, which mirrors the two extremes found in the MEP data - the highest facilitation with visual palm down imagery and lowest with kinesthetic palm up imagery.

There are several possible explanations for the failure to find higher facilitation during kinesthetic imagery. First, it is difficult to manipulate kinesthetic information without sufficient familiarity with the task constraints. Behavioral evidence indicates that mental practice incorporating kinesthetic imagery is useful to athletes with expertise in a task, but neither aids nor hinders novices [20]; and, people feigning injury incorrectly anticipate the effect of the impairment on real and imagined movement [30]. Task unfamiliarity would allow few parameters with which to make a predictive movement to be centralized accurately. If the efferent information related to kinesthetic parameters is incorrect or unknown, it should follow that the motor system tries to minimize the information. Thus, the failure of kinesthetic palm up imagery to induce facilitation with respect to static imagery; participants could not accommodate incongruent predictive information (initial sensory information of the hand and motor outflow [cf. 44]), and therefore the motor system suppressed or ignored the conflicting information [cf. 55]. Indeed, the thumb-palm opposition movement is one which people are likely to spend little time consciously monitoring and in this sense is relatively unfamiliar.

Moreover, the modality of an image may be less important than its purpose. While it is frequently assumed that motor imagery is simply the image of an action, this overlooks the fact that motor imagery may serve several functions. Athletes use imagery for cognitive functions (rehearsal of skills or strategies) and motivational functions (goal obtainment, manipulating arousal, self-confidence or mental toughness) $[18,36]$. Motivational functions also accompany exercise imagery (feeling energized or reducing stress, physical appearance, exercise self-efficacy and positive emotions) [15], with many of these 
functions related to initiating and sustaining exercise behavior. Thus, motor imagery is not simply an image of an action but a highly dynamic cognitive process that may be heavily influenced by motivational aspects of behavior. Motor imagery used for motivational purposes should be able to generate changes (e.g. respiration or muscle tension) in the peripheral nervous system.

A TMS paper considering the effects of visual and kinaesthetic imagery showed modulation of excitability between the $\mathrm{ON}$ and OFF phases of kinesthetically imagined movement; by contrast, the visual motor imagery comparison failed to reach significance [52]. The seeming discrepancy between this paper and our present results may lie in the protocol of the two experiments. While we used a stimulation intensity of $120 \% \mathrm{rMT}$, Stinear et al. [52] adjusted the intensity to "... produce responses of approximately $1.0 \mathrm{mV}$ amplitude in the resting APB (p. 3)". Using this procedure implied that different stimulation intensities were used in different subjects. Moreover, the Stinear et al. stimulation intensity may therefore have been well above ours; based on our data, we would have needed to stimulate far above $120 \%$ in order to have used the $1.0 \mathrm{mV}$ criteria. This is important because increases in stimulation intensity lead to the activation of corticospinal neurons with higher thresholds. A recent study [54] using six stimulation intensities (range 100-150\% rMT) illustrates the importance of this point.

The present study demonstrates that research on motor imagery can and should be carefully controlled. The use of the post experimental manipulation check enabled the identification and replacement of participants who did not abide by the instructions. The analysis of the feedback allows us to exclude explanations of the results based on differences in subjective experience. Most critically, the absence of clear differences between imagery modalities highlights a problem underlying a large number of motor imagery studies. Specifically, unless subjective information regarding the imagery actually experienced is obtained, one cannot make decisive statements based on corticospinal excitability to claim which imagery modality measured.

In conclusion, we found no evidence that first person visual and kinesthetic imagery leads to different levels of corticospinal excitability per se. We have found evidence that excitability fluctuates with congruency, particularly when the imagery was kinesthetic.

\section{Acknowledgement}

This research was supported by grants MIUR and FIRB awarded to Salvatore M. Aglioti.

\section{Appendix A}

\section{A.1. Kinesthetic imagery (e.g. with palm up)}

Raise your right hand with the palm facing up and perform continuous thumb-palm opposition. Make the movement accurately. Notice how the movement "feels". You select the frequency of the movement. This frequency must be constant.
Continue this action for $10 \mathrm{~s}$ while observing your hand perform the movement. Now imagine yourself performing the movement using first person imagery. The first person imagery should be egocentric. Imagine the "feel" of the movement (i.e. the muscle tension and stretch)

\section{A.2. Visual imagery (e.g. with palm down)}

Raise your right hand with the palm facing down and perform continuous right thumb-palm opposition. Make the movement accurately. You select the frequency of the movement. This frequency must be constant. Continue this action for $10 \mathrm{~s}$ while observing your hand perform the movement. Now imagine yourself performing the movement using first person imagery. The first person imagery should be egocentric. Your imagery should contain only that visual information which was available to you when you were watching the real movement. Do not imagine the "feel" of the movement.

At the start of each trial the computer will emit a beep sound. This sound is the signal to begin your imagery. Continue to perform the imagery with your eyes closed. The imagined movement should have the same frequency as the real movement. After several seconds a TMS pulse will be delivered. At this point you should stop your imagery and verbally report the direction your imagined thumb was moving (left or right) when the TMS pulse was delivered. After several seconds the computer will emit a beep sound indicating the start of another trial.

\section{References}

[1] Abruzzese G, Trompetto C, Schieppati M. The excitability of the human motor cortex increases during execution and mental imagination of sequential but not repetitive finger movements. Exp Brain Res 1996;111:465-72.

[2] Atienza F, Balaguer I, Garcia-Merita ML. Factor analysis and reliability of the movement imagery questionnaire. Percept Motor Skills 1994;78:1323-8.

[3] Bonnet M, Decety J, Jeannerod M, Requin J. Mental simulation of an action modulates the excitability of spinal reflex pathways in man. Cogn Brain Res 1997;5:221-8.

[4] Briggs GG, Nebes RD. Patterns of hand preference in a student population. Cortex 1975;11:230-8.

[5] Chen R, Classen J, Gerloff C, Celnik P, Wassermann EM, Hallet M, et al. Depression of motor cortex excitability by low-frequency transcranial magnetic stimulation. Neurology 1997;48:1398-403.

[6] Chua R, Evans JT. What the hand can't tell the eye: illusion of space constancy during accurate pointing. Exp Brain Res 2005;162:109-14.

[7] Cohen J. A power primer. Psychol Bull 1992;112:155-9.

[8] Dunlap WP, Cortina JM, Vaslow JB, Burke MJ. Meta-analysis of experiments with matched groups or repeated measures designs. Psychol Methods 1996;1:170-7.

[9] Ersland L, Ròsen G, Lundervold A, Smievoll AI, Tillung T, Sundberg H, et al. Phantom limb imagery fingertapping causes primary motor cortex activation: an fMRI study. Neuroreport 1996;8:207-10.

[10] Facchini S, Meullbacher W, Battaglia F, Boroojerdi B, Hallett M. Focal enhancement of motor cortex excitability during motor imagery: a transcranial magnetic stimulation study. Acta Neurol Scand 2002;105:146-51.

[11] Feltz DL, Landers DL. The effects of mental practice on motor skill learning and performance: a meta-analysis. J Sport Psychol 1983;5:25-57. 
[12] Fourkas A, Avenanti A, Urgesi C, Aglioti S. Corticospinal excitability during first and third person imagery. Exp Brain Res 2006;68:143-51.

[13] Fourkas AD, Martenuik RG, Khan MA. Guiding movements with internal representations: a reach-and-grasp task. Res Q Exerc Sport 2003;74:165-72.

[14] Gerardin E, Sirigu A, Lehéricy S, Poline JP, Gaymard B, Marsault C, et al. Partially overlapping neural networks for real and imagined hand movements. Cereb Cortex 2000;10:1093-104.

[15] Giacobbi PR, Hausenblas HA, Fallon EA, Hall CA. Even more about exercise imagery: a grounded theory of exercise imagery. J Appl Sport Psychol 2003;15:160-75.

[16] Hale BD. The effects of internal and external imagery on muscular and ocular concomitants. J Sport Psychol 1982;4:379-87.

[17] Hale BS, Raglin JS, Koceja DM. Effect of mental imagery of a motor task on the Hoffmann reflex. Behav Brain Res 2003;142:81-7.

[18] Hall CR, Mack D, Paivio A, Hausenblas H. Imagery use by athletes: development of the Sport Imagery Questionnaire. Int J Sport Psychol 1998;29:73-89.

[19] Hall CR, Martin KA. Measuring movement imagery abilities: a revision of the movement imagery questionnaire. J Ment Imagery 1997;21:143-54.

[20] Hardy L, Callow N. Efficacy of external and internal visual imagery perspectives for the enhancement of performance on tasks in which form is important. J Sport Exerc Psychol 1999;21:95-112.

[21] Hashimoto R, Rothwell JC. Dynamic changes in corticospinal excitability during motor imagery. Exp Brain Res 1999;125:75-81.

[22] Jacobson E. Electrical measurements of neuromuscular states during mental activities: V, variation of specific muscles contracting during imagination. Am J Physiol 1931;96:115-21.

[23] Jacobson E. Electrophysiology of mental activities. Am J Psychol 1932;44:677-94.

[24] Jamieson S. Likert scales: how to (ab)use them. Med Educ 2004;38:1217-8.

[25] Johnson P. The functional equivalence of imagery and movement. Q J Exp Psychol 1982;34A:349-65.

[26] Johnson SH, Sprehn G, Saykin AJ. Intact motor imagery in chronic upper limb hemiplegics: evidence for activity-independent action representations. J Cogn Neurosci 2002;14:841-52.

[27] Kasai T, Kawai S, Kawanishi M, Yahagi S. Evidence for facilitation of motor evoked potentials (MEPs) induced by motor imagery. Brain Res 1997;744:147-50.

[28] Lotze M, Montoya P, Erb M, Hülsmann E, Flor H, Klose U, et al. Activation of cortical and cerebellar motor areas during executed and imagined hand movements: an fMRI study. J Cogn Neurosci 1999;11:491-501.

[29] Lutz RS. Covert muscle excitation is outflow from the central generation of motor imagery. Behav Brain Res 2003;140:149-63.

[30] Maruff P, Velakoulis D. The voluntary control of motor imagery: imagined movements in individuals with feigned motor impairment and conversion disorder. Neuropsychologia 2000;38:1251-60.

[31] Morris SB, DeShon RP. Combining effect size estimates in meta-analysis with repeated measures and independent-groups designs. Psychol Methods 2002;7:105-25.

[32] Murphy SM, Jowdy DP. Imagery and mental practice. In: Horn TS, editor. Advances in sport psychology. Champaign, IL: Human Kinetics; 1992. p. $221-50$

[33] Naito E, Kochiyama T, Kitada R, Nakamura S, Matsumura M, Yonekura $\mathrm{Y}$, et al. Internally simulated movement sensations during motor imagery activate cortical motor areas and the cerebellum. J Neurosci 2002;22:3683-91.

[34] Oakley DA, Whitman LG, Halligan PW. Hypnotic imagery as a treatment for phantom limb pain: two case reports and a review. Clin Rehab 2002;16:368-77.

[35] Osborne J. Notes on the use of data transformations. Pract Assess Res Eval, 2002;8:http://PAREonline.net/getvn.asp?v=8\&n=6.

[36] Paivio A. Cognitive and motivational functions of imagery in human performance. Can J Appl Sport Sci 1985;10:22S-8S
[37] Pascual-Leone A, Grafman J, Hallet M. Modulation of cortical motor output maps during development of implicit and explicit knowledge. Science 1994;263:1287-9.

[38] Parsons LM, Fox PT, Downs JH, Glass T, Hirsch TB, Martin CC, et al. Use of implicit motor imagery for visual shape discrimination as revealed by PET. Nature 1995;375:54-8.

[39] Petit LS, Pegna AJ, Mayer E, Hauert CA. Representation of anatomica constraints in motor imagery: mental rotation of a body segment. Brain Cogn 2003;51:95-101.

[40] Porro CA, Francescato MP, Cettolo V, Diamond ME, Baraldi P, Zuian $\mathrm{C}$, et al. Primary motor and sensory cortex activaton during motor performance and motor imagery: a functional magnetic resonance imaging study. J Neurosci 1996;16:7688-98.

[41] Rossini PM, Rossi S, Pasqaletti P, Tecchio F. Corticospinal excitability modulation to hand muscles during movement imagery. Cereb Cortex 1999;9:161-7.

[42] Roth M, Decety J, Raybaudi M, Massarelli R, Delon-Martin C, Segebarth $\mathrm{C}$, et al. Possible involvement of primary motor cortex in mentally simulated movement: a functional magnetic resonance imaging study. Neuroreport 1996;7:1280-4.

[43] Schnitzler A, Salenius S, Salamin R, Jousmäki V, Hari R. Involvemen of primary motor cortex in motor imagery: a neuromagnetic study. Neuroimage 1997;6:201-8.

[44] Schwoebel J, Boronat CB, Branch Coslett H. The man who executed "imagined" movements: evidence for dissociable components of the body schema. Brain Cogn 2002;50:1-16.

[45] Schwoebel J, Friedman R, Duda N, Coslett HB. Pain and the body schema: evidence for peripheral effects on mental representations of movement. Brain 2001;124:2098-104.

[46] Shenton JT, Schwoebel J, Coslett HB. Mental motor imagery and the body schema: evidence for proprioceptive dominance. Neurosci Lett 2004;370:19-24.

[47] Sirigu A, Duhamel JR. Motor and visual imagery as two complementary but neurally dissociable mental processes. J Cogn Neurosci 2001;13:910-9.

[48] Sirigu A, Duhamel JR, Cohen L, Pillon B, Dubois B, Agid Y. The mental representation of hand movements after parietal cortex damage. Science 1996;273:1564-8.

[49] Solodkin A, Hlustik P, Chen EE, Small SL. Fine modulation in network activation during motor execution and motor imagery. Cereb Cortex 2004;14:1246-55.

[50] Stephan KM, Fink GR, Passingham RE, Silbersweig D, CeballosBaumann AO, Frith CD, et al. Functional anatomy of the mental representation of upper extremity movements in healthy subjects. J Neurophysiol 1995;73:373-86.

[51] Stevens JA. Interference effects demonstrate distinct roles for visual and motor imagery during the mental representation of human action Cognition 2005;95:329-50

[52] Stinear CM, Byblow WD, Steyvers M, Levin O, Swinnen SP. Kinesthetic, but not visual, motor imagery modulates corticospinal excitability. Exp Brain Res 2006;168:157-64.

[53] Stinear CM, Byblow WD. Motor imagery of phasic thumb abduction temporally and spatially modulates corticospinal excitability. Clin Neurophysiol 2003;114:909-14.

[54] Takahashi M, Hayashi S, Ni Z, Yahagi S, Favilla M, Kasai T. Physica practice induces excitability changes in human hand motor area during motor imagery. Exp Brain Res 2005;163:132-6.

[55] Vargas CD, Olivier E, Craighero L, Fadiga L, Duhamel JR, Sirigu A. The influence of hand posture on corticospinal excitability during motor imagery: a transcranial magnetic stimulation study. Cereb Cortex 2004:14:1200-6.

[56] Wassermann EM. Risk and safety of repetitive transcranial magnetic stimulation: report and suggested guidelines from the International Workshop on the Safety of Repetitive Transcranial Magnetic Stimulation, June 5-7, 1996. Electroencephalogr Clin Neurophysiol 1998;108: $1-16$ 